\title{
Interview questions for Dr. Divya Khosla
}

Submitted Aug 28, 2018. Accepted for publication Sep 14, 2018.

doi: $10.21037 / \mathrm{atm} .2018 .10 .58$

View this article at: http://dx.doi.org/10.21037/atm.2018.10.58

\section{Expert's introduction}

Dr. Divya Khosla (Figure 1) is a tenured Assistant Professor in the Department of Radiation Oncology at the prestigious Postgraduate Institute of Medical Education and Research (PGIMER), Chandigarh. She has experience of more than 10 years in this field. Her expertise is mainly in the field of gastrointestinal, breast and lung malignancies. In addition to being a faculty in an academic institute, she is also an invited faculty at various national conferences. She is an active member of several professional organisations and has numerous publications to her credit which includes original articles, invited reviews and chapters in the book.

\section{Interview}

ATM: You have contributed an article entitled "Concurrent therapy to enhance radiotherapeutic outcomes in glioblastoma" to ATM. You have mentioned in the article that "The molecular biology of glioblastoma is complex and poses treatment challenges." What are the challenges? Is there any solution to these challenges?

Dr. Khosla: Glioblastoma is the most challenging malignancy to treat as the survival remains dismal despite multiple advancements in treatment. The molecular biology of Glioblastomas is complex due to heterogeneity at morphological, molecular and genetic levels. These molecular and genetic alterations result in alteration of cellular transduction pathways, changes in cellular metabolism, loss of cell cycle control, marked angiogenesis and genetic instability. The intertumoral and intratumoral heterogeneity hinders in the development of effective treatment as it is difficult to categorise patients for inclusion into trials and treatment. The key signaling pathways that contribute to the pathogenesis of disease include $\mathrm{p} 53, \mathrm{pRb}$, PI3K-PTEN-Akt-mTOR and RAS/MAPK pathways. The majority of clinical trials evaluating therapies targeting these commonly mutated or deregulated signaling pathways in GBM have shown little to no clinical efficacy. These tumors exhibit significant genomic instability leading to therapeutic

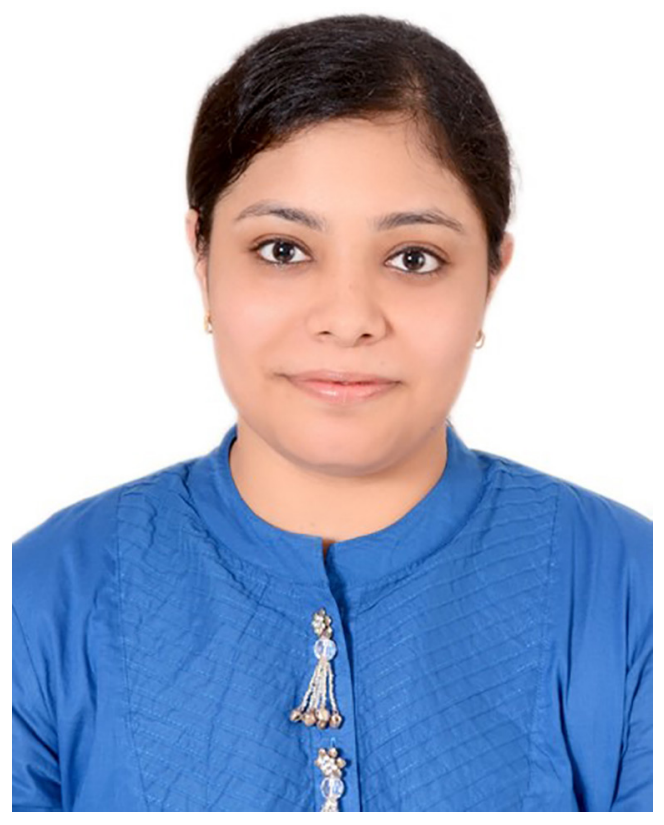

Figure 1 Dr. Divya Khosla.

resistance to various single targeted agents. In order to achieve better results in glioblastomas, multiple pathways need to be targeted simultaneously to develop effective treatment.

\section{ATM: You mentioned in your article that "Addition of valproic acid to standard treatment may improve the outcome but needs to be validated in further studies." Does any application of valproic acid in this field improve the treatment of glioblastomas in the past two years?}

Dr. Khosla: Valproic acid is widely used as an antiepileptic in glioblastoma patients. Various retrospective studies have shown improved survival outcome with valproic acid in glioblastomas. It inhibits histone deacetylase resulting in radiosensitization. A pooled analysis of survival association of antiepileptic drug (valproic acid) use at the start of chemoradiotherapy with temozolomide was performed in the four randomized clinical trials in newly diagnosed 
glioblastoma patients: AVAGlio, CENTRIC, CORE, and Radiation Therapy Oncology Group 0825. The use of valproic acid was not associated with any benefit in progression free survival or overall survival. However, more prospective studies on larger samples are needed for validation of results.

\section{ATM: What's the future outlook of the treatment of glioblastomas?}

Dr. Khosla: The treatment of glioblastomas is one of the most challenging tasks in oncology. Despite various therapeutic interventions, the prognosis remains dismal and cure remains elusive. Use of temozolomide has been the only major and successful advancement in the management of glioblastomas. Molecular profiling has become important part of the standard neuropathological evaluation of glioblastomas. Due to molecular heterogeneity, multiple combined approaches targeting multiple pathways are required to achieve success in treatment. Studies evaluating novel therapeutic approaches that combine newer targeted therapies with standard treatments are warranted. There is need to develop personalized rational treatment strategies with individually tailored therapy based on molecular and genomic heterogeneity to overcome the heterogeneous nature of GBM.

ATM: How did you become involved in this research of glioblastomas, and bow would you describe the particular challenges, setbacks, and successes you've encountered along the way?

Dr. Khosla: As we see large number of these patients at our institute and it is challenging to treat these patients which

Cite this article as: Zheng ES. Interview questions for Dr. Divya Khosla. Ann Transl Med 2018;6(23):465. doi: 10.21037/ atm.2018.10.58 led me to write an article on it. The current standard of care is maximal safe surgical resection followed by adjuvant radiotherapy with concomitant and adjuvant temozolomide. The major challenge in managing glioblastomas is that recurrence is inevitable. Though multiple novel therapies are available but these therapies need to be used in conjunction with the current standard of care, highlighting the aggressive and heterogeneous nature of this tumor. We, at our institute give concurrent and adjuvant temozolomide with radiation therapy. As other therapies are investigational, more research needs to be carried out to establish effective treatment to improve outcome in these patients.

ATM: What do you think would be important factors for a paper to be informative (liked by peers)?

Dr. Khosla: Review article which gives an overview about certain topic in brief by compiling all the research together is highly regarded. The article should provide valuable insight into the nature of disease, pathogenesis, available treatment modalities, standard of care, newer treatment strategies, novel drugs and ongoing research.

\section{Acknowledgements}

None.

\section{Footnote}

Conflicts of Interest: The author has no conflicts of interest to declare.

(Science Editor: Elva S. Zheng, ATM, editor@atmjournal.org) 